University of Wollongong

Research Online

Australian Institute for Innovative Materials -

Papers

Australian Institute for Innovative Materials

$1-1-2015$

Injectable phenytoin loaded polymeric microspheres for the control of temporal lobe epilepsy in rats

Jonathan Jiang

University of Melbourne

Zhilian Yue

University of Wollongong, zyue@uow.edu.au

Sebastien Bauquier

University of Melbourne

Alan Lai

University of Melbourne

Yu Chen

University of Wollongong, yc456@uowmail.edu.au

See next page for additional authors

Follow this and additional works at: https://ro.uow.edu.au/aiimpapers

Part of the Engineering Commons, and the Physical Sciences and Mathematics Commons

Research Online is the open access institutional repository for the University of Wollongong. For further information contact the UOW Library: research-pubs@uow.edu.au 


\title{
Injectable phenytoin loaded polymeric microspheres for the control of temporal lobe epilepsy in rats
}

\begin{abstract}
Epilepsy is a prevalent neurological disorder with a high frequency of drug resistance. While significant advancements have been made in drug delivery systems to overcome anti-epileptic drug resistance, efficacies of materials in biological systems have been poorly studied. The purpose of the study was to evaluate the anti-epileptic effects of injectable poly(epsiloncaprolactone) (PCL) microspheres for controlled release of an anticonvulsant, phenytoin (PHT), in an animal model of epilepsy. Methods: PHT$P C L$ and Blank-PCL microspheres formulated using an oil-in-water (O/W) emulsion solvent evaporation method were evaluated for particle size, encapsulation efficiency, surface morphology and in-vitro drug release profile. Microspheres with the most suitable morphology and release characteristics were subsequently injected into the hippocampus of a rat tetanus toxin model of temporal lobe epilepsy. Electrocorticography (ECoG)from the cerebral cortex were recorded for all animals. The number of seizure events, severity of seizures, and seizure duration were then compared between the two treatment groups. Results: We have shown that small injections of drug-loaded microspheres are biologically tolerated and released PHT can control seizures for the expected period of time that is in accord with invitro release data. Conclusion: The study demonstrated the feasibility of polymer-based delivery systems in controlling focal seizures.
\end{abstract}

\section{Keywords}

lobe, epilepsy, rats, loaded, injectable, polymeric, phenytoin, microspheres, control, temporal

\section{Disciplines}

Engineering | Physical Sciences and Mathematics

\section{Publication Details}

Jiang, J. L., Yue, Z., Bauquier, S. H., Lai, A., Chen, Y., McLean, K. J., Halliday, A. J., Sui, Y., Moulton, S., Wallace, G. G. \& Cook, M. J. (2015). Injectable phenytoin loaded polymeric microspheres for the control of temporal lobe epilepsy in rats. Restorative Neurology and Neuroscience, 33 (6), 823-834.

\section{Authors}

Jonathan Jiang, Zhilian Yue, Sebastien Bauquier, Alan Lai, Yu Chen, Karen J. McLean, Amy J. Halliday, Yi Sui, Simon E. Moulton, Gordon G. Wallace, and Mark J. Cook 


\section{Injectable phenytoin loaded polymeric microspheres for the control of temporal lobe epilepsy in rats}

Jonathan L. Jiang ${ }^{\mathrm{a}, \mathrm{b}, *}$, Zhilian Yue ${ }^{\mathrm{c}}$, Sebastien H. Bauquier ${ }^{\mathrm{d}}$, Alan Lai ${ }^{\mathrm{a}}$, Yu Chen ${ }^{\mathrm{c}}$, Karen J. McLean ${ }^{\mathrm{a}, \mathrm{b}}$, Amy J. Halliday $^{\mathrm{a}}$, Yi Sui ${ }^{\mathrm{e}}$, Simon Moulton ${ }^{\mathrm{c}}$, Gordon G. Wallace ${ }^{\mathrm{c}}$ and Mark J. Cook ${ }^{\mathrm{a}, \mathrm{b}}$ a St Vincent's Department of Medicine, University of Melbourne, Fitzroy, VIC, Australia ${ }^{\mathrm{b}}$ Centre for Clinical Neurosciences and Neurological Research, St. Vincent's Hospital Melbourne, Fitzroy, VIC, Australia

${ }^{c}$ Intelligent Polymer Research Institute and ARC Centre of Excellence for Electromaterials Science, AIIM Facility, Innovation Campus, University of Wollongong, Wollongong, NSW, Australia ${ }^{\mathrm{d}}$ Faculty of Veterinary Science, University of Melbourne, 250 Princes Hwy, Werribee, VIC, Australia

e Department of Neurology, Shenyang First People's Hospital, Shenyang, Liaoning, China (PRC)

Abstract.

Purpose: Epilepsy is a prevalent neurological disorder with a high frequency of drug resistance. While significant advancements have been made in drug delivery systems to overcome anti-epileptic drug resistance, efficacies of materials in biological systems have been poorly studied. The purpose of the study was to evaluate the anti-epileptic effects of injectable poly(epsiloncaprolactone) (PCL) microspheres for controlled release of an anticonvulsant, phenytoin (PHT), in an animal model of epilepsy. Methods: PHT-PCL and Blank-PCL microspheres formulated using an oil-in-water $(\mathrm{O} / \mathrm{W})$ emulsion solvent evaporation method were evaluated for particle size, encapsulation efficiency, surface morphology and in-vitro drug release profile. Microspheres with the most suitable morphology and release characteristics were subsequently injected into the hippocampus of a rat tetanus toxin model of temporal lobe epilepsy. Electrocorticography (ECoG)from the cerebral cortex were recorded for all animals. The number of seizure events, severity of seizures, and seizure duration were then compared between the two treatment groups. Results: We have shown that small injections of drug-loaded microspheres are biologically tolerated and released PHT can control seizures for the expected period of time that is in accord with in-vitro release data.

Conclusion: The study demonstrated the feasibility of polymer-based delivery systems in controlling focal seizures.

Keywords: Biodegradable, polymer, drug delivery, epilepsy, phenytoin, microspheres

\section{Introduction}

Advances in biomaterial science over the past decades have resulted in a dramatic expansion in the

\footnotetext{
*Corresponding author: Dr. Jonathan L. Jiang, St Vincent's Department c 3065, Australia. Tel. +61 39288 3517; Fax: +61 392883350 E-mail: jonathan.jiang@unimelb.edu.au.
}

use of polymer-based drug delivery systems (Halliday \& Cook, 2009; Halliday et al., 2012). In addition to applications in management of cancers and diabetes (Gallia et al., 2005; Traitel et al., 2000), multiple animal studies have demonstrated successes in using polymer based implants to treat neurological disorders including Parkinson's disease, Huntington's disease and Alzheimer's disease (Halliday \& Cook, 2009). 
Unfortunately, limited attempts have been made in the use of these novel biomaterials in animal models of epilepsy (Halliday et al., 2013).

Epilepsy is one of the most prevalent serious neurological disorders, affecting over 50 million people globally (de Boer et al., 2013). Despite its severity, approximately one-third of people with epilepsy fai to respond to anti-epileptic drugs (AEDs) and continue to experience seizure episodes (Sander, 2003; Shorvon 1996). It has been recognized that after the failure of two first-line AEDs, the chance of achieving seizure freedom with additional therapeutic regimens drops as low as $5-10 \%$ (Kwan \& Brodie, 2000).

If seizures are unsatisfactorily controlled using AEDs, then non-pharmacological therapies are often considered. Epilepsy surgery represents a viable option in appropriately selected refractory candidates, where the seizure origin in the brain can be localized, and where the lesion region is safe for resection. The success of inducing long-lasting seizure remission from epilepsy surgery range from a low $25 \%$ for patients who have extra hippocampal seizure origin, to $70 \%$ in appropriately selected candidates (Kwan \& Sperling, 2009). The decision to undergo surgery require individual based risk-benefit analysis, and not al patients with medically refractory epilepsy are suitable candidates for surgery. Given that there are limited alternative treatment modalities, new therapies are urgently required.

While a great deal is known about seizures and epilepsy, little is known about the mechanisms responsible for the high frequency of drug resistance. The main concern with AED therapy is the bioavailability of the medication at the epileptic focus, as refractory patients tend to develop systemic and cerebral side effects before a large enough dose of the medication can be taken to ameliorate seizures (Schmid \& Loscher, 2005). Consequently, it is argued that focal drug delivery method in epilepsy would allow medications to by-pass the blood-brain-barrier thereby achieving high levels of drugs in the brain, while main taining low levels of drugs in the body to avoid systemic side effects.

A polymer based intracranial implantable device that is capable of releasing AEDs over time represents one approach to focal drug delivery. Early studie on polymer-based drug delivery devices for the treatment of epilepsy focused mainly on non-biodegradable polymers in the form of ethylene-vinyl acetate (EVA) rods and pellets (Boison et al., 1999; Kokaia et al.
1994; Tamargo et al., 2002). Some of these materials showed sustained drug release and implantation in animal systems demonstrated positive seizure control in multiple studies. In comparison, biodegradable polymers used in neurological disorders are typically made of poly (lactide-co-glycolide) (PLGA), polycaprolactone (PCL), or polylactic acid (PLA) in the form of films or injectable microspheres (Giunchedi et al., 1998; Halliday et al., 2013; Li et al., 2007). Our recent in-vivo study using levetiracetam loaded PLGA sheets demonstrated a temporary reduction in seizure duration following implantation above the motor cortex in an animal model of focal epilepsy (Halliday et al., 2013). However, the antiepileptic effect was limited as the subdural polymer sheet implants were placed some distance from the seizure focus. An injectable microsphere delivery system using biodegradable polymers should be better suited for the treatment of focal epilepsy, particularly when seizures originate in the less accessible hippocampal regions, the most common site of human focal seizure onset.

Injectable particulate formulations characterized by microspheres and nanospheres have been generated using a variety of techniques including, the emulsion-evaporation method, spray-drying and electro-spraying. Research to date has been heavily focused on improving release kinetics of particulate formulations. Unfortunately, few studies have tested the efficacy of the microspheres in-vivo. PCL-based implants are very well tolerated by biological systems (Ghorab et al., 2011; Marchal-Heussler et al., 1993) and given their biocompatibility we sought to investigate the efficacy with the anticonvulsant drug phenytoin (PHT). PHT was chosen because it is an effective anticonvulsant used for monotherapy of partial seizures and has been found to be superior to the newer AEDs in its anticonvulsant action (Kwan \& Brodie, 2003).

In this article, process and formulation variables influencing the preparation of PHT-loaded PCL microspheres by emulsion-evaporation were studied. Once physical characterization of microspheres and drug release studies were completed, the drug-loaded microspheres were implanted in a rat model of temporal lobe epilepsy induced through intra-hippocampal injection of tetanus toxin. Tetanus toxin is secreted by the bacterium Clostridium tetani. When injected directly into the brain, tetanus toxin acts to block the inhibitory neurons and thereby produces epileptic foci centred on the injection site. In the current study, targeting the toxin 
to hippocampus we have generated a model of complex partial seizures (temporal lobe or limbic epilepsy) (Schwartzkroin et al., 2006). Compared to other animal models, tetanus toxin produces recurrent spontaneous epileptic seizures that do not have major cell loss as an early component of the syndrome and does not start with status epilepticus (prolonged epileptic crisis).

\section{Materials and methods}

\subsection{Animals}

Eight-weeks old male Sprague-Dawley rats were obtained from the Animal Resources Centre (WA, Australia) and housed individually with access to food and water. All experiments were approved by the $\mathrm{St}$ Vincent's Hospital (Melbourne) Animal Ethics Committee and conducted in accordance with the Australian Code of Practice for the Care and Use of Animals for Scientific Purposes (2004).

\subsection{Polymer sphere production}

PHT-loaded microspheres were prepared by a modified $\mathrm{O} / \mathrm{W}$ emulsion-solvent evaporation method (Barbato et al., 2001). Briefly, PCL (molecular weight $80,000 \mathrm{Da}$ ) and PHT were dissolved in $10 \mathrm{~mL}$ of dichloromethane and acetone (dichloromethane : acetone $=8: 2)$ at a weight ratio of $20: 1$. The concentration of PCL varied from $3 \% \mathrm{w} / \mathrm{v}, 6 \% \mathrm{w} / \mathrm{v}, 7.5 \% \mathrm{w} / \mathrm{v}, 9 \%$ $\mathrm{w} / \mathrm{v}, 12 \% \mathrm{w} / \mathrm{v}$ and $15 \% \mathrm{w} / \mathrm{v}$. Each organic solution was emulsified into $100 \mathrm{~mL}$ of $2 \% \mathrm{w} / \mathrm{v}$ poly-vinyl alcohol (PVA) aqueous solution at $7000 \mathrm{rpm}$ for 3 min using an IKA ${ }^{\circledR}$ T25 digital ULTRA-TURRAX ${ }^{\circledR}$ homogenizer. PVA was used to stabilize the emulsion formed between polymer and drug solution with water and to reduce particle aggregation (Jenkins \& Harrison, 2006). Then $100 \mathrm{~mL}$ of deionized water was added to the $\mathrm{O} / \mathrm{W}$ emulsion, and the mixture was stirred at $1000 \mathrm{rpm}$ at room temperature for $4 \mathrm{~h}$. The microspheres were isolated by centrifugation at $2500 \mathrm{rpm}$ for $5 \mathrm{~min}$ and then washed multiple times with $0.2 \%$ w/v PVA solution at $37^{\circ} \mathrm{C}$ to remove any residual drug crystals. After a final wash with deionized water, the microspheres were dried initially under a stream of $\mathrm{N}_{2}$ and then placed in a vacuum oven at $37^{\circ} \mathrm{C}$ overnight. They were stored at $-20^{\circ} \mathrm{C}$ prior to physiochemical characterization and animal studies. Drug-free (blank) microspheres were also prepared using a similar procedure as described above.

\subsection{Characterization of microspheres}

Morphology of the microspheres was examined using a field emission scanning electron microscope (FESEM, JEOL JSM-7500FA). The samples were sputter-coated with gold to avoid sample charging. Mean particle sizes and size distribution were quantified from photomicrographs taken using a Zeiss Axiovert 40 CFC microscope. Images of each polymer microsphere sample were then analysed using NIH ImageJ software. Distributions of particle diameter were obtained by evaluating at least 50 particles per sample.

\subsection{Loading efficiency}

The amount of PHT loading per weight of microspheres was determined by a solvent extraction method. Microspheres were weighed and extracted with ethanol overnight. The extract was collected by centrifugation at $10,000 \mathrm{rpm}$ for $10 \mathrm{~min}$ and the amount of PHT quantified by a modified HPLC method (Lin et al., 2010). An Agilent 1260 Infinity HPLC system was employed, equipped with an Atlantis ${ }^{\circledR} \mathrm{T} 3 \mathrm{C} 18 \mathrm{col}-$ umn $(250 \mathrm{~mm} \times 4.6 \mathrm{~mm}, 5 \mu \mathrm{m})$. The temperature of the $\mathrm{C} 18$ column was controlled at $40^{\circ} \mathrm{C}$, the injection volume was $10 \mu \mathrm{L}$, the flow rate $0.8 \mathrm{~mL} / \mathrm{min}$, and UV detection wavelength at $203 \mathrm{~nm}$. The isocratic mobile phase was composed of water and acetonitrile (50:50, v/v). A standard curve of PHT prepared in artificial cerebrospinal fluid (aCSF) was established by plotting the peak areas against respective concentrations of standard solutions.

2.5. In-vitro drug release study

The PHT-loaded microspheres $(10 \mathrm{mg})$ were suspended in $1 \mathrm{~mL}$ of aCSF, and the release experiment was conducted at $37^{\circ} \mathrm{C}$ in a shaker water bath. For each sample, the release medium solution was collected by centrifugation and replenished with fresh aCSF at various time points, and stored at $-20^{\circ} \mathrm{C}$ prior to HPLC analysis being undertaken.

\subsection{Stereotaxic surgery and $E C o G$ recording}

In-vivo study comprises a two-stage surgery. First, tetanus toxin (Calbiochem, USA) is administered 
unilaterally to the hippocampus of adult rats to induce temporal lobe epilepsy. The animals were then given 14 days for the development of generalized seizure events, before the administration of either drug-loaded or blank microspheres.

In detail, rats were anaesthetized with an i.p. injection of ketamine $(70 \mathrm{mg} / \mathrm{kg})$ and xylazine $(10 \mathrm{mg} / \mathrm{kg}$ immediately prior to surgery. Carprofen $(5 \mathrm{mg} / \mathrm{kg}$, s.c.) was also administered for pain relief. Once anaesthetized, the rats were placed in a stereotaxic apparatus, and isoflurane $(0.5 \%$ in oxygen, $2 \mathrm{~L} / \mathrm{min})$ was administered via a nose-cone. A small trephine opening was drilled over the right hippocampi at coordinates $3.5 \mathrm{~mm}$ caudal to Bregma and $3.0 \mathrm{~mm}$ away from the midline using the atlas of Paxinos and Watson (Paxinos \& Watson, 2007). Using a Hamilton microsyringe and infusion pump (KD Scientific Inc., USA) $125 \mathrm{~nL}$ of tetanus toxin solution per $100 \mathrm{~g}$ of body weight was injected at a rate of $100 \mathrm{~nL} / \mathrm{min}$ into the right hippocampal CA3 area. Tetanus toxin solution contained $25 \mu \mathrm{g}$ of tetanus toxin dissolved in $250 \mu \mathrm{L}$ of $0.05 \mathrm{M}$ phosphate buffered saline (PBS; Sigma-Aldrich, UK) and $2 \%$ bovine serum albumin (Sigma-Aldrich, UK). The syringe was left in-situ for $5 \mathrm{~min}$ before being slowly retracted to avoid back flow through the injection track Following the injection, five screw electrodes were placed epidurally at five positions on the skull and a $23 \mathrm{G}$ guide-cannula was placed over the top of the trephine opening (Fig. 1). Electrodes and cannula were fixed to the skull using dental acrylic and the openended cannula covered with surgical tape. Following surgery, animals were placed on heat pads for recovery, housed in single cages and allowed to recover for four days.

Synchronized video-electrocorticography (ECoG) monitoring was commenced on Day 5, and continued until the end of the experiments. ECoG was sampled at $2,048 \mathrm{~Hz}$ and recorded using Grael HD PSG / EEG Amplifier (Compumedics, Australia) and ProFusion EEG 4 software package (Compumedics, Australia). ECoG recordings were necessary to determine seizure duration as well as identifying sub-clinical events. Video was recorded synchronously using a digital network-camera (Vivotek, Taiwan).

\subsection{Polymer microsphere implantation}

Following nine days of ECoG recording, the animals were randomly removed from recording cages at Day 14 and anesthetized with an i.p. injection of ketamine $(70 \mathrm{mg} / \mathrm{kg})$ and xylazine $(10 \mathrm{mg} / \mathrm{kg})$. Once anaesthetized, rats were placed back on stereotaxic apparatus and $0.5 \%$ isoflurane in oxygen were administered via nose-cone. Openings to the cannulas were uncovered and $2 \mu \mathrm{L}$ of either Blank-PCL $(n=6)$ or PHT-PCL $(n=6)$ loaded microspheres $(1.5 \mu \mathrm{g}$ of spheres dispersed in $2 \mu \mathrm{L}$ of saline) were injected at a rate of $150 \mathrm{~nL} / \mathrm{min}$. Microspheres made from $9 \% \mathrm{PCL}$ were used as they demonstrated satisfactory loading efficiency and suitable morphology. Following microsphere implantation, the syringe was left in place for $10 \mathrm{~min}$ before being slowly retracted to avoid back flow through the injection track. Cannula openings were then sealed with dental acrylic and wounds sutured. The animals were allowed to recover on heat pads and placed back in recording cages for further ECoG monitoring. To monitor recovery from the second surgery animals were taken out from the recording cage and weighed daily. In addition, exploratory activity and grooming behaviour were monitored twice daily (also recorded on $24 \mathrm{hrs}$ video recording).
A

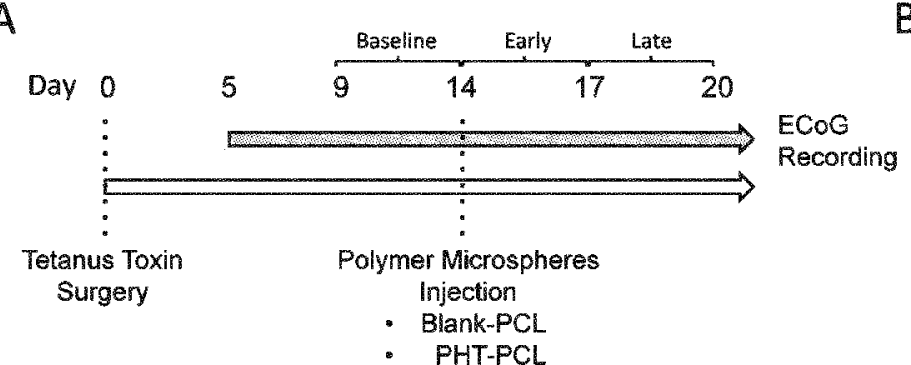

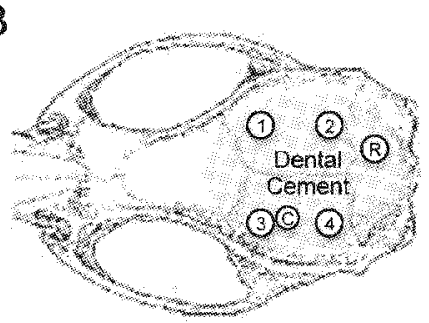

Fig. 1. Experiment Design (A); Schematic diagram illustrating the positioning of four epidural recording screw electrodes, Reference Electrode ${ }^{\circledR}$ and Cannula $\mathfrak{O}(\mathrm{B})$. 


\subsection{Data analysis}

Recorded ECoGs were visualized using ProFusion EEG 4 software package (Compumedics, Australia). For each animal, 6-hours of ECoG recordings $(6 \mathrm{pm}$ $12 \mathrm{am}$ ) were manually annotated for seizure onset (marked by the initiation of continuous spike wave discharge that is greater than $5 \mathrm{~s}$ in duration) until six days after the implantation of polymer microspheres. Due to inter-subject variation in seizure frequencies and release data obtained from in-vitro study, seizure duration and number of seizure events were averaged over
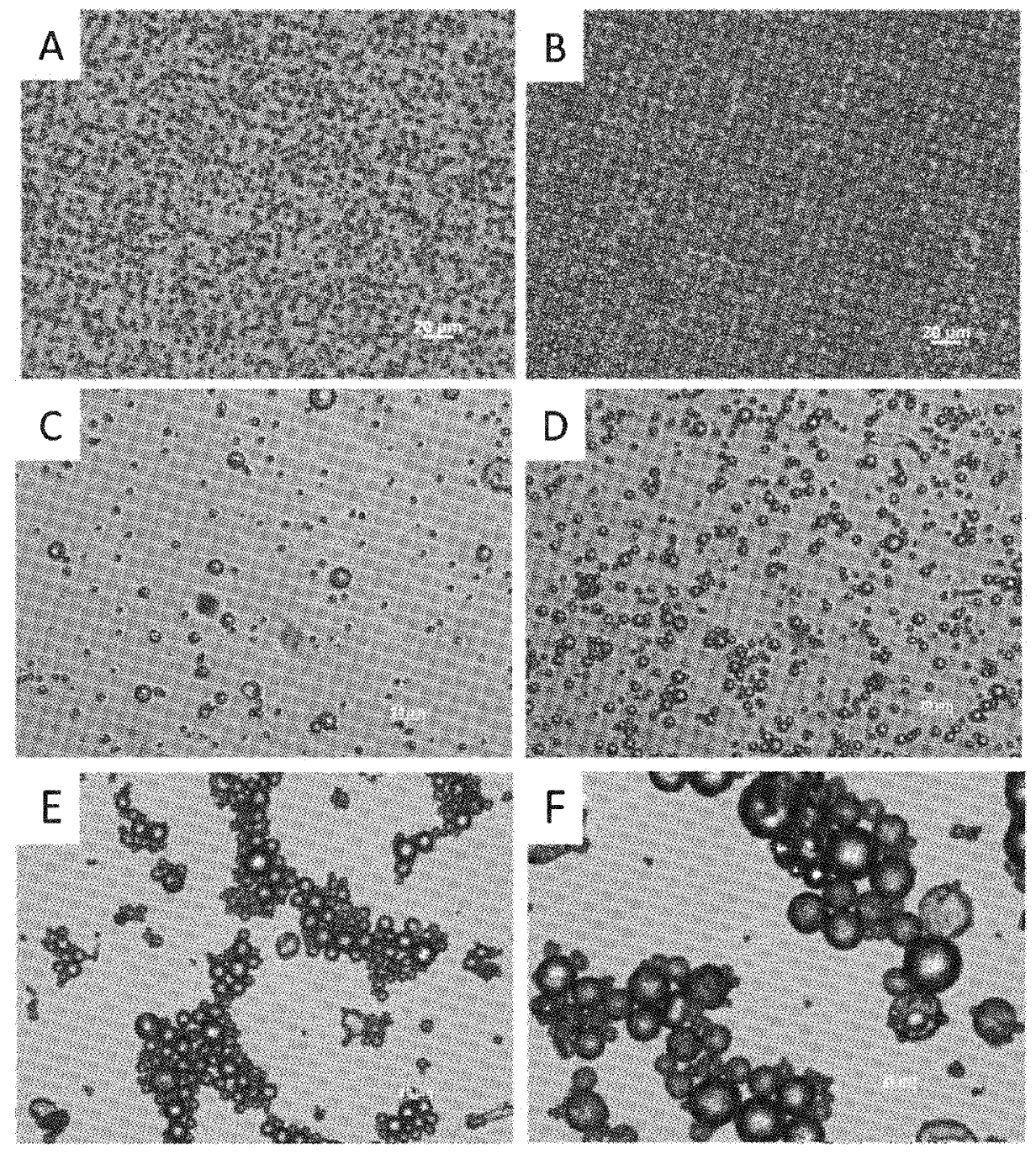

Fig. 2. Photomicrographs of the spheres prepared using an $\mathrm{O} / \mathrm{W}$ emulsion from (A) $3 \%$, (B) $6 \%$, (C) $7.5 \%$, (D) $9 \%$,(E) $12 \%$ and (F) $15 \%$ PCL.

three day blocks. The first three days following microsphere implantation is termed the 'Early' monitoring block where the next three days was designated as the 'Late' monitoring block. The duration of each seizure was defined as the time (in seconds) from the beginning of polyspike activity to the termination of spike and wave activity prior to the post-ictal depression. The mean seizure duration for each block were calculated by (a) first averaging the duration of all seizures that a rat had in a single recording session (6 hours), (b) then averaging all that rat's session means over the three day block, and (c) determining the mean \pm the
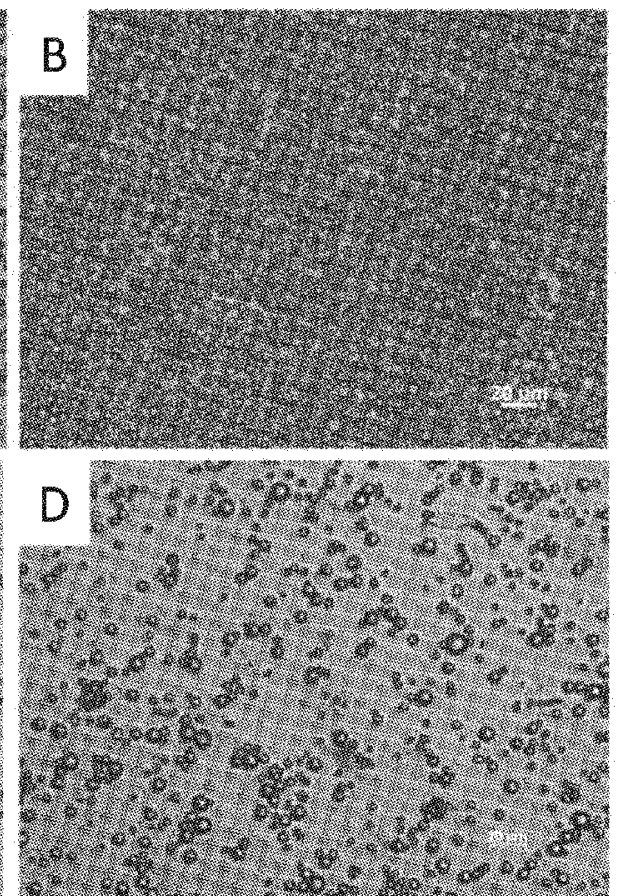
standard error of the mean (S.E.M) of the block averages from all rats.

Severity of detected electrographic seizures were classified using modified Racine Stages based on synchronous video recording (Racine, 1972): where stage 1 is manifested as behavioural arrest with mouth/facia movements; stage 2 , head nodding; stage 3 , forelimb clonus; stage 4 , rearing; stage 5 , rearing and falling; stage 6, loss of posture and generalized tonic clonic seizure activity. For simplicity, in this study, seizures were classified either as Non-Convulsive Seizures (correlating to Racine Stage 1-3) or Convulsive seizures (Correlating to Racine stage 4-6).

\subsection{Statistical analysis}

Results were statistically analysed using Sigma Stat (v11 0, Systat Software, San Jose, CL, USA) and GraphPad Prism (GraphPad Software, CA, USA) software. Differences in the median seizure duration and frequency between Blank-PCL and PHT-PCL treated groups were determined using Two-Way ANOVA with Repeated Measure. All results are shown as mean \pm S.E.M. unless otherwise stated.

\section{Results}

\subsection{Characteristics of PHT-loaded spheres}

PHT-loaded PCL microspheres were successfully prepared by an $\mathrm{O} / \mathrm{W}$ emulsion solvent evaporation method. Samples prepared with $3 \%$ and $6 \%$ PCL resulted in the formation of mono dispersed micro sphere dispersions. Once the polymer concentration was increased beyond $7.5 \%$, the associated increase in PHT concentration resulted in concurrent formation of large drug crystals with microspheres. Since drug crystals complicate the drug release profile, they were removed with an additional overnight washing step at $37^{\circ} \mathrm{C}$ (Fig. 2)

We have found that increased polymer concentration correlated with desirable increases in drug loading efficiency and that the particle size of the spheres increased with a broader distribution (Table 1). A higher polymer concentrations (12\% w/v and $15 \%$ PC w/v preparations), the microspheres agglomerated and formed clusters as shown in Fig. 2 (E) and (F).

Based on this characterization, a 9\% PCL preparation was chosen for in-vivo administration as it offered
Table 1

Summary of microsphere characteristion at different PCL concentrations

\begin{tabular}{lrcc}
\hline Samples & $\begin{array}{c}\text { Particle Size } \\
(\mu \mathrm{m})\end{array}$ & $\begin{array}{c}\text { Drug loading } \\
\text { efficiency }(\%)\end{array}$ & $\begin{array}{c}\text { Drug loading } \\
\text { efficiency }(\%) \\
\text { after o/n wash }\end{array}$ \\
\hline 3\% PCL & $4.69 \pm 0.05$ & $4.2 \pm 0.2$ & - \\
6\% PCL & $4.33 \pm 0.04$ & $23.2 \pm 1.8$ & - \\
$7.5 \%$ PCL & $6.75 \pm 0.36$ & $30.7 \pm 3.3$ & $12.5 \pm 3.3$ \\
$9 \%$ PCL & $7.29 \pm 0.11$ & $53.1 \pm 7.5$ & $39.3 \pm 3.2$ \\
$12 \%$ PCL & $6.48 \pm 0.85$ & $45.5 \pm 2.2$ & $29.5 \pm 0.8$ \\
$15 \%$ PCL & $17.72 \pm 3.39$ & $83.9 \pm 9.6$ & $54.5 \pm 3.9$ \\
\hline
\end{tabular}

small particles for smooth injection and high encapsulation efficiency, which are important in minimizing tissue damage while maintaining sufficient drug loading. SEMs of the 9\% PCL microspheres confirmed its spherical structure with smooth surface morphology (Fig. 3).

PHT release from the 9\% PCL preparation was investigated in $\mathrm{aCSF}(\mathrm{pH} 7.4)$ solution at $37^{\circ} \mathrm{C}$ for 30 days. Following the overnight wash to remove undissolved drug crystal, PHT release from the PCL microsphere samples showed multi-stage release behaviour, as shown in Fig. 4. During the first 2 days, release was linear with about $80 \mu \mathrm{g}$ of PHT released per day. In the following 2 days, the release rate decreased to about $20 \mu \mathrm{g} /$ day before reaching $90 \%$ completion at day 4. Studies have shown that the degradation of PCL is known to follow an erosion mechanism, which is very slow in aqueous medium due to its semicrystallinity and hydrophobic structure (Cho et al., 1999; Jeong et al., 2003). This view supports the argument that the above drug release is predominantly a result of diffusion.

\subsection{Electrocorticography $(E C o G)$}

Unilateral injection of tetanus toxin into the hippocampus resulted in a syndrome of spontaneous seizures previously described (Mellanby et al., 1977). Rats that received tetanus toxin began to exhibit repeated Wet Dog Shake (WDS) behaviour pattern at approximately day 5-6 following toxin injection. As limbic seizures progressed toward generalization, high frequency epileptiform discharges recorded on the ECoG would increase in duration, and clinica events would progress from non-convulsive episodes of behavioural arrest, staring and chewing (between day 7-9 following toxin injection) to convulsive seizure events involving behavioural arrest, facial 


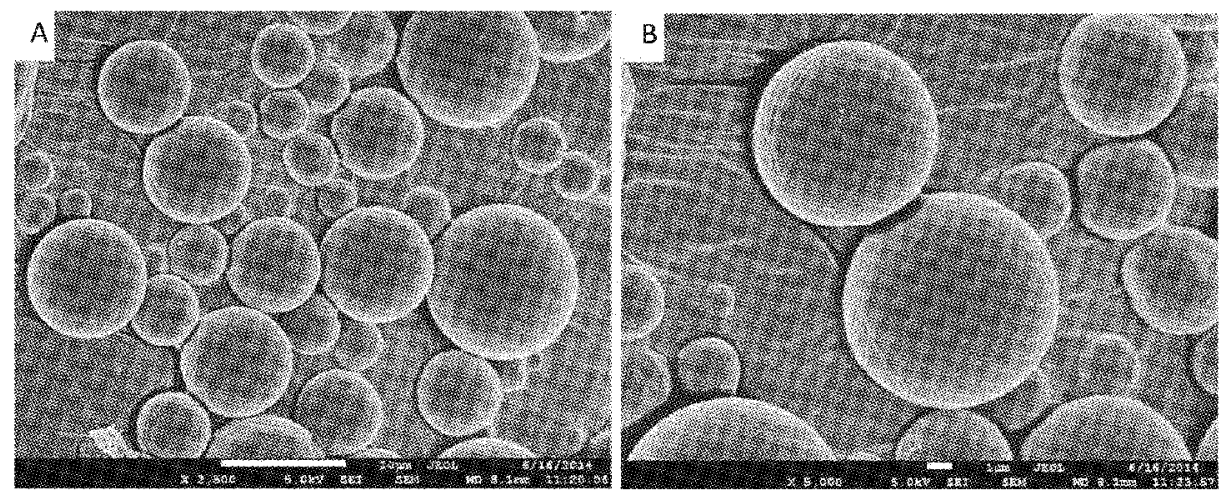

Fig. 3. SEM micrographs of the PHT-loaded microspheres prepared from 9\% w/v PCL using an O/W emulsion method taken at $\times 2,5000$ and $\times 5,000$ magnification.
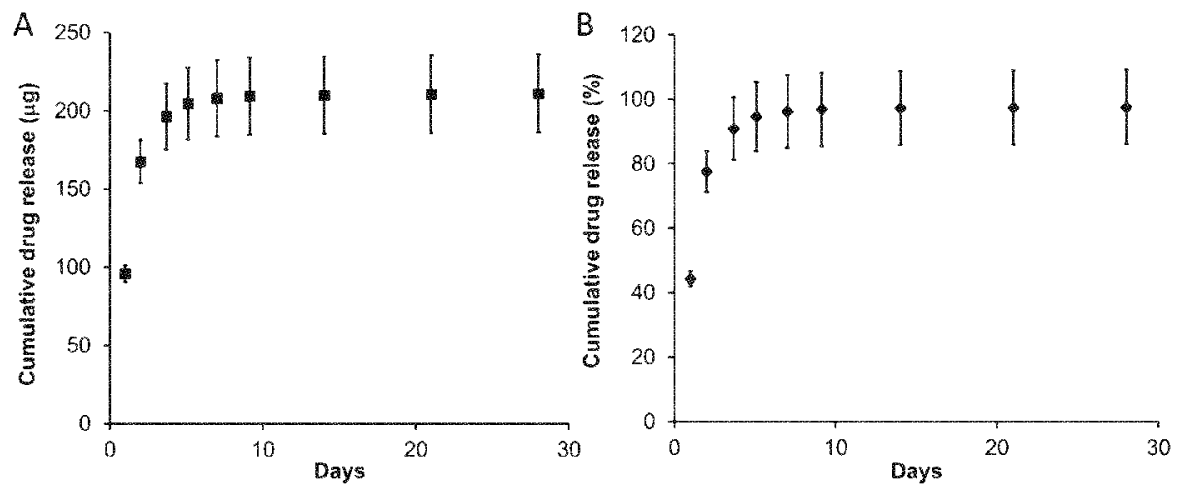

Fig. 4. In-vitro release profiles of drug from the PHT loaded PCL microspheres in aCSF pH 7.4 at $37^{\circ} \mathrm{C}$. The PHT loaded PCL microspheres were prepared from $9 \% \mathrm{w} / \mathrm{v}$ PCL using an O/W emulsion method.

clonus, bilateral forelimb clonus, rearing and falling after Day 9 for most subjects. The duration of each seizure was measured using ECoG and was defined as the time (in seconds) from the beginning of polyspike activity to the termination of spike and wave activity prior to the post-ictal depression (Fig. 5).

\subsection{Overall seizure number and duration}

As the majority of drug release was within the first three days, effects of microsphere implantation were categorized into 'Early' (within 3 days) and 'Late' (after 3 days) monitoring blocks. All animals recovered from the microsphere injection with no recorded reductions in body weights. Furthermore, no signs of protest or stress were identified with animals receiving the microsphere injection demonstrating normal exploratory and grooming behaviour.Immediately after the implantation of PHT-PCL polymer microspheres, rats demonstrated reduced number of seizure events in the Early monitoring block compared to those that received blank polymer spheres (Blank-PCL $14.78 \pm 2.803$; PHT-PCL $5.233 \pm 1.625 ; P=0.0146$ ) Similarly, total seizure duration was decreased in the Early monitoring block in rats implanted with PHTPCL polymers (Blank-PCL 577.0 \pm 79.05 seconds; PHT-PCL: $192.4 \pm 61.51$ seconds; $P=0.0033$ ). The anti-epileptic effect did not extend beyond the initial three days as there were no statistically significant differences between the groups in the Late-monitoring block (Fig. 6).

\subsection{Seizure severity}

Seizures were further categorized either as convulsive or non-convulsive. We found mean duration 
A

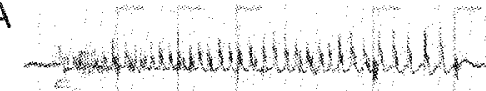

B

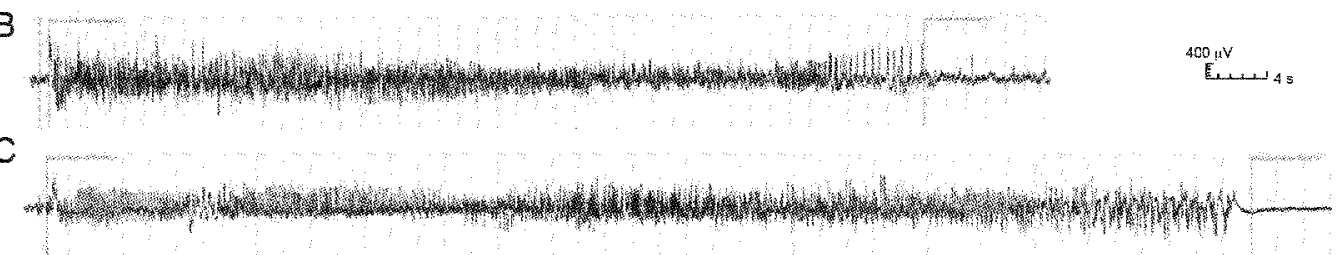

Fig. 5. Typical electrocorticograms recorded from a tetanus-toxin injected rat showing the development of epilepsy from Wet Dog Shake (A) to Non-convulsive seizure (B), and to Convulsive Seizure (C). Both non-convulsive and convulsive seizures showed initial phase of high frequency polyspike activity of increasing amplitude followed by lower amplitude rhythmic spike and wave activity.
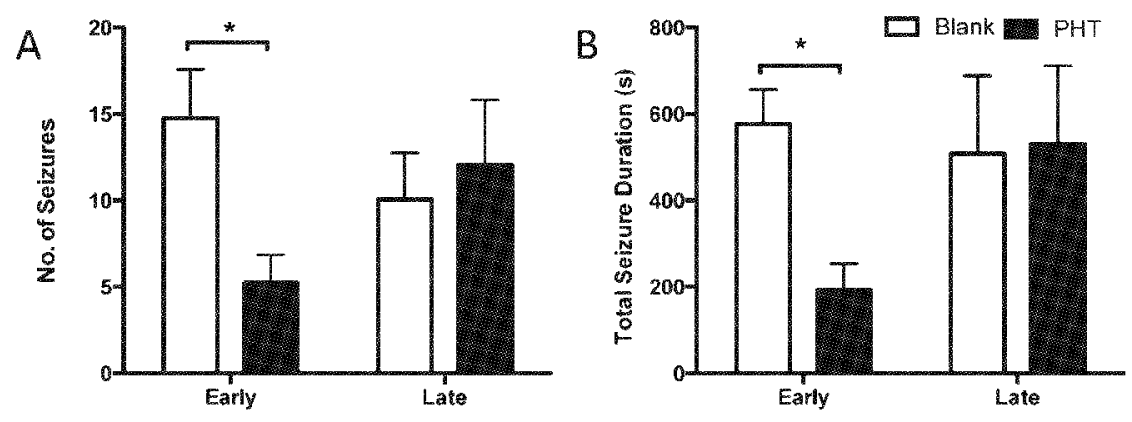

Fig. 6. Graphs showing injection of PHT-loaded microspheres significantly reducedthe number of seizure events (A), and total seizure duration (B) in the Early monitoring period compared to the control (Blank) group. No change was detected in the Late monitoring group.

of a single convulsive and non-convulsive seizures were not significantly affected by focal PHT delivery (Fig. 7A and B). Furthermore, no significant antiepileptic effect was found on convulsive seizures, as the number of convulsive seizures (Fig. 7C) and total convulsive seizure duration (Fig. 7D) remain unchanged following PHT injection. In comparison, an anti-epileptic effect was identified for nonconvulsive seizures. In the Early monitoring block there was a trend towards fewer seizures in PHT-PCL implanted rats (Fig. 7E). However, while the effect was not statistically significant (Blank $=12.17 \pm 3.533$, PHT $=3.832 \pm 1.733 ; \quad P=0.06 ; \quad$ Fig. $7 \mathrm{E}$ ), a significant decrease in total non-convulsive seizure duration was observed $($ Blank $=438.0 \pm 114.8 \mathrm{~s}$, $\mathrm{PHT}=121.5 \pm 56.91 \mathrm{~s}, P=0.0331$; Fig. $7 \mathrm{~F}$ ). Again, this anti-epileptic effect did not extend to the Late monitoring block. Collectively, the result suggests focal PHT treatment has more therapeutic effect on preventing the initiation of non-convulsive seizure but once the seizure is initiated it has no influence on seizure propagation

\section{Discussion}

Limitations with systemic drug administration such as systemic toxicity and sub-optimal bioavailability in pathological loci have led to a dramatic increase in the research of polymer-based drug delivery. While significant advancements have been made, applications of materials in functional biological systems have somewhat lagged behind its development, especially in the field of epilepsy research where systemic drug administration has been found ineffective for about one-third of the patients. The systemic effects of the medications, including hepatic enzyme induction, osteoporosis, and teratogenicity are increasingly recognized as major clinical problems (Kwan \& Brodie, 2000).

We have demonstrated that a single injection of PHT- releasing microspheres into a rat model of temporal lobe epilepsy resulted in significant reduction in epileptic events with no observed clinical side effects. The time course of the improvement was also consistent with in-vitro release characteristics of PHT-PCL microspheres. This study shares 

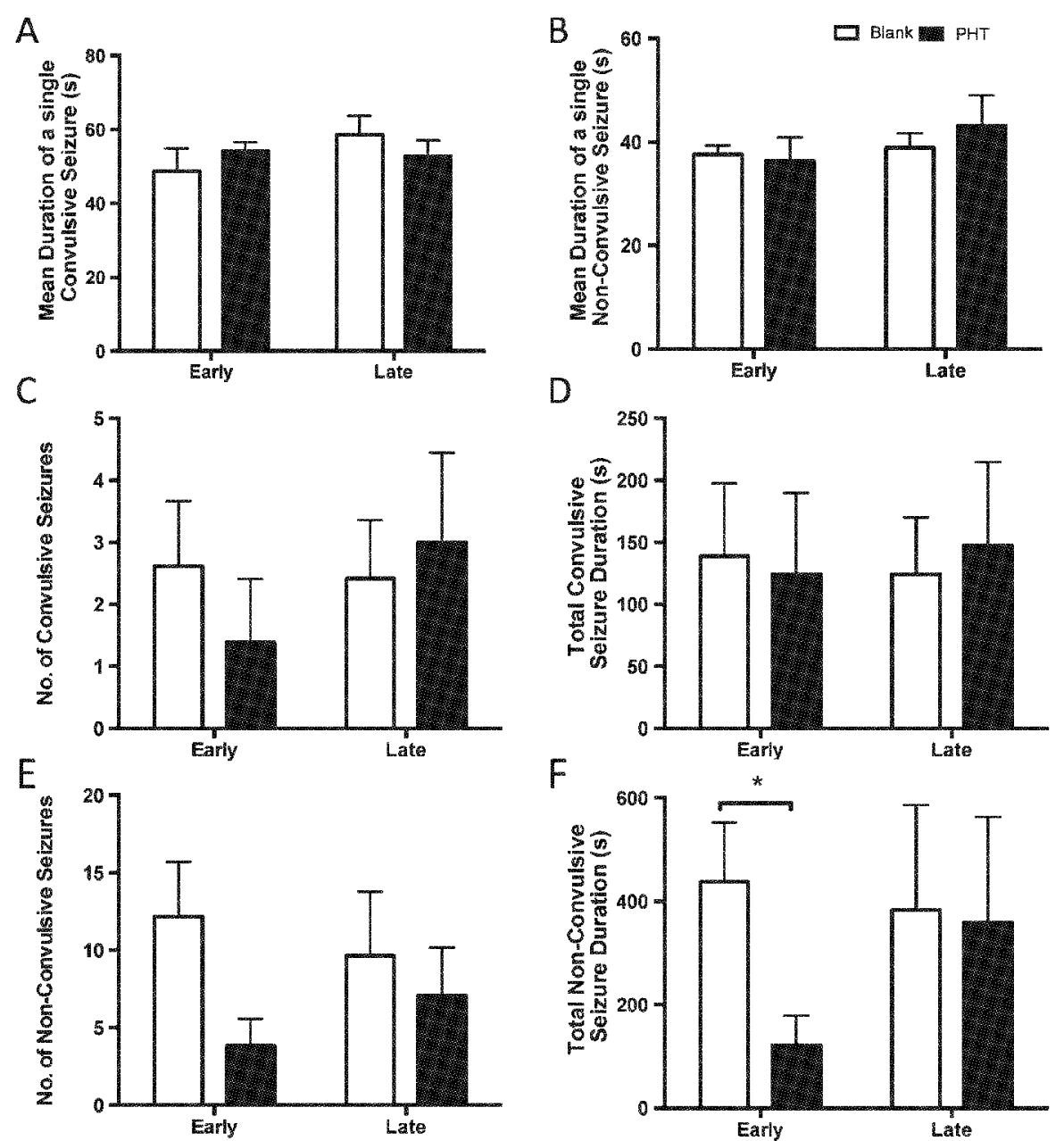

Fig. 7. Graphs showing administration of PHT-loaded polymer microspheres does not influence mean duration of a single convulsive (A), or non-convulsive (B) event. Similarly, focal PHT delivery has no effect on the number of convulsive events (C) or total convulsive seizure duration
(D). The reduction in the number of non-convulsive seizures in the PHT group was not statistically significance in the Early monitoring block (E). However, the total non-convulsive seizure duration in the Early monitoring block was significantly decreased in the PHT group (F). No (E). However, the total non-convulsive seizure du

some similarity with tetanus toxin-induced neocortical epilepsy models, where seizures respond positively to focal delivery of NBQX (2,3-dihydroxy-6-nitro-7sulfamoyl-benzo(F)-quinoxaline) but were refractory to systemic administration of PHT and diazepam (Nilsen et al., 2005). The mechanism for the drug resistance was not elaboratein Nilsen's study, but others have reported a 30 -fold reduction in extracellular PHT concentration in the hippocampus compared to available serum concentration following intravenous injection (Wang \& Patsalos, 2003). Consequently, if systemic PHT resistance is shared between the models, findings from the current study would support the argument that drug resistance is due to the lack of effective penetration into brain parenchyma as the concentration of PHT released locally from the microspheres $(\sim 20-30 \mu \mathrm{mol} / \mathrm{L})$ would be significantly higher than the level normally achieved with systemic administration (Loscher, 2005).

For targeted drug delivery to be clinically acceptable, it needs to 1) exert minimal mechanical damage when administered to biological systems, 2) distribute 
away from the site of injection to cover epileptogenic sites that are more diffused than the current focal lesion, and 3) achieve satisfactory long-term release to avoid repetitive injection.

Mechanical damage is created through surgical insertion of a needle into brain tissue as well as size of injected microspheres. We found there were significan correlations among polymer concentration, loading efficiency and particle size. As with other studies, loading efficiency increased with higher concentration of polymer constituent as it promotes rapid solidification of particles (Jeong et al., 2003; Yeo \& Park, 2004). While increases in loading efficiency were desirable, it must be balanced by undesirable increase in particle size as it directly affects the size of needle used for surgery. Previous studies on intracerebral delivery of PHT used Ethylene Vinyl Acetate Copolymer (EVAc) polymer implants measured $5 \mathrm{~mm}$ in diameter and $4 \mathrm{~mm}$ in height (Tamargo et al., 2002). In comparison, microspheres injected in the current study were significantly smaller and with a uniform size distribution they were small enough to pass freely through the $26 \mathrm{G}$ Hamilton syringes, which has been used extensively in rodent stereotaxic surgeries with few reports of nonspecific mechanic damage (Fornari et al., 2012; Hosak et al., 2013). From a practical standpoint, we have also found larger particles were increasingly prone to rapid rate of sedimentation in the vehicle solution, which would result in blockage of the injector as well as fluctuation in injected concentration.

Determination of epileptogenic site would follow the typical presurgical evaluation, which can include: neurological and neuropsychological exams, High Density EEG / High Density EEG-MEG, EEG-fMRI, PET, interictal and ictal SPECT, and some times invasive intracranial recordings. While accuracy is difficult to quantify, non-invasive high density EEG typically reported to localize down to $2-6 \mathrm{~cm}^{2}$ in cortex. MEG is less affected by tissue and can localize down to les than $1 \mathrm{~cm}^{2}$, but has a drop off in terms of sensitivity for deeper brain structures (Malmivuo et al., 1997 Plummer et al., 2008, 2010). In the current study we did not evaluate whether the distribution of injected microspheres would satisfactorily cover more diffused epileptogenic sites typically seen in clinical scenarios, as what seemed more relevant is the distribution of the released drug. A small molecular drug such as PHT, once released from the spheres, may diffuse quickly into the surrounding tissue, especially when the turnover rate of cerebrospinal fluid in the brain is fairly rapid. Hence, although epileptogenic site may not be identified as a precise focal lesion in clinical settings, once drug concentration and volume are titrated, it would be reasonable to expect small molecular AEDs to cover the pathological locus.

In the current study, an $\mathrm{O} / \mathrm{W}$ solvent evaporation method was used for the preparation of PCL microspheres as it represented a simple technique for the encapsulation of lipophillic drugs. However, a disadvantage of preparing microspheres using solvent evaporation methods is the potential presence of residual organic solvents in the final product (Lee et al. 2008). In the current study the drug loaded microspheres were subjected to an overnight washing step in order to remove insoluble PHT drug crystals as well as the residual solvent. Typically drug release from biodegradable spheres can be achieved by degradation of polymer matrix and/or diffusion of drug in both matrix and reservoir type microspheres (Varde \& Pack, 2004). Degradation of PCL polymer in-vivo should be very slow. It has been reported by Seyednejad et al that PCL should remain intact within 3 month of implantation (Seyednejad et al., 2012), and in a separate study by Sun et al, PCL capsules remained intact in shape during 2-year implantation (Sun et al., 2006). As formulated microspheres released the majority of loaded drug in the first $48-72 \mathrm{hrs}$ following implantation, it suggests that the drug was released by diffusion rather than polymer degradation. Consequently, antiepileptic effect observed in the current study was limited. Future research would be focused on generating drug-loaded microspheres with more sustained release profile to reduce the frequency of 'repetitive administration'. This can be achieved by either altering the encapsulation paradigm or investigate 'smart polymers' that change surface morphology depending on intracellular environment (Buchsbaum et al. 2014; Liechty et al., 2010). These stimuli-responsive polymer systems would either respond to local calcium/sodium concentration changes or coupled with implantable seizure detection/stimulation devices and releases content by physical stimulus. Either approach would reduce the need for repeated intracranial administration, thereby making the approach clinically acceptable.

Our study outlines one such proof of concept trial where focal AED delivery specifically to the seizure focus could potentially provide seizure relief in cases of refractory epilepsy. Nevertheless, further investigations are required to examine long-term seizure control 
by using particle formulations with more sustained release profiles.

\section{Conclusion}

In this study we show for the first time that bioengineered delivery of PHT can promote significant seizure reductions in an animal model of temporal lobe epilepsy. We have demonstrated that biodegradable microspheres are a suitable, minimally invasive approach for drug resistant epileptic patients. These findings encourage further research in polymer drug delivery systems in the management of epilepsy.

\section{Acknowledgments}

The authors thank the Australian Research Council and National Health \& Medical Research Council for continuing financial support. The authors gratefully acknowledge the sponsorship of the Victorian Government through its Science Technology and Innovation Initiative administered by the Department of Industry, Innovation and Regional Development. GGW thanks the ARC for his Laureate Fellowships. The authors also acknowledge use of the facilities and the assistance of Mr. Tony Romeo at the UOW Electron Microscopy Centre. The authors are grateful for access to materials synthesis and characterization equipment made available through the Australian National Fabrication Facility (ANFF)

\section{References}

Barbato, F., La Rotonda, M.I., Maglio, G., Palumbo, R., \& Quaglia. F. (2001). Biodegradable microspheres of novel segmented poly(ether-ester-amide)s based on poly(epsilon-caprolactone) for the delivery of bioactive compounds. Biomaterials, 22(11), 1371-1378.

Boison, D. Scheurer, L. Tseng, J.L. Aebischer, P., \& Mohler, H. (1999). Seizure suppression in kindled rats by intraventricular grafting of an adenosine releasing synthetic polymer. $E x p$ lar grafting of an adenosing
Neurol, I60(1), 164-174.

Cho, C.S., Han, S.Y., Ha, J.H., Kim, S.H., \& Lim, D.Y (1999). Clonazepam release from bioerodible hydrogels based on semi-interpenetrating polymer networks composed of poly(epsilon-caprolactone) and poly(ethylene glycol) macromer. Int J Pharm, 181(2), 235-242.

de Boer, H.M., Moshe, S.L., Korey, S.R., \& Purpura, D.P. (2013). ILAE/IBE/WHO Global Campaign Against Epilepsy: A partILAE/IBE/WHO Global Campaign Against Epilepsy: A
nership that works. Curr Opin Neurol, 26(2), 219-225.
Fornari, R.V., Wichmann, R., Atsak, P., Atucha, E., Barsegyan, A., Beldjoud, H., Roozendaal, B., et al., (2012). Rodent stereotaxic surgery and animal welfare outcome improvements for

Gallia, G.L., Brem, S., \& Brem, H. (2005). Local treatment of malignant brain tumors using implantable chemotherapeutic polymers. J Natl Compr Canc Netw, 3(5), 721-728.

Ghorab, D.M., Amin, M.M., Khowessah, O.M., \& Tadros, M.I (2011). Colon-targeted celecoxib-loaded Eudragit(R) S100coated poly-epsilon-caprolactone microparticles: Preparation, characterization and in vivo evaluation in rats. Drug Deliv, 18(7), 523-535.

Giunchedi, P., Conti, B., Scalia, S., \& Conte, U. (1998). In vitro degradation study of polyester microspheres by a new HPLC
method for monomer release determination. J Control Release. $56(1-3), 53-62$.

Halliday, A.J., Campbell, T.E., Nelson, T.S., McLean, K.J., Wallace, G.G., \& Cook, M.I. (2013). Levetiracetam-loaded biodegradable polymer implants in the tetanus toxin model of temporal lobe epilepsy in rats. J Clin Neurosci, 20(1), 148-152.

Halliday, A.J., \& Cook, M.J. (2009). Polymer-based drug delivery devices for neurological disorders. CNS Neurol Disord Drug Targets, 8(3), 205-221.

Halliday, A.J., Moulton, S.E., Wallace, G.G., \& Cook, M.J. (2012). Novel methods of antiepileptic drug delivery - polymer-based implants. Adv Drug Deliv Rev, 64(10), 953-964.

Hosaka, K., Downes, D.P., Nowicki, K.W., \& Hoh, B.L. (2013). Modified murine intracranial aneurysm model: Aneurysm formation and rupture by elastase and hypertension. J Neurointerv mation and rupture by
Surg, 6(6), 474-479.

Jenkins, M.J., \& Harrison, K.L. (2006). The effect of molecular weight on the crystallization kinetics of polycaprolactone. Polymers for Advanced Technologies, 17(6), 474-478.

Jeong, J.C., Lee, J., \& Cho, K. (2003). Effects of crystalline microstructure on drug release behavior of poly(epsilon-
caprolactone) microspheres.J Control Release, 92(3), 249-258.

Kokaia, M., Aebischer, P., Elmer, E., Bengzon, J., Kalen, P., Kokaia, Z., \& Lindvall, O. (1994). Seizure suppression in kindling epilepsy by intracerebral implants of GABA- but not by noradrenaline-releasing polymer matrices. Exp Brain Res, 100(3), 385-394.

Kwan, P., \& Brodie, M.J. (2000). Early identification of refractory epilepsy. N EngI J Med, 342(5), 314-319.

Kwan, P., \& Brodie, M.J. (2003). Clinical trials of antiepileptic medications in newly diagnosed patients with epilepsy. Neurology, 6O(11 Suppl 4), S2-S12.

Kwan, P., \& Sperling, M.R. (2009). Refractory seizures: Try additional antiepileptic drugs (after two have failed) or go directly to early surgery evaluation? Epilepsia, 5or Suppl 8), 57-62.

Lee, J., Oh, S., Joo, M.K., \& Jeong, B. (2008). Solvent-free preparation of caprolactone oligomer microspheres. Journal of Physics and Chemistry of Solids, 69(5-6), 1596-1599.

Li, Z., Li, Q., Simon, S., Guven, N., Borges, K., \& Youan, B.B. (2007). Formulation of spray-dried phenytoin loaded poly(epsilon-caprolactone) microcarrier intended for bran 
Lin, P.C., Hsieh, Y.H., Liao, F.F. \& Chen, S.H. (2010). Determination of free and total levels of phenytoin in human plasma from tion of free and total levels of phenytoin in human plasma from
patients with epilepsy by MEKC: An adequate alternative to patients with epilepsy by MEKC: An aded
HPLC. Electrophoresis, 31(9), 1572-1582.

Loscher, W. (2005). Mechanisms of drug resistance. Epileptic Disord, 7(Suppl 1), S3-S9.

Malmivuo, J., Suihko, V., \& Eskola, H. (1997). Sensitivity distributions of EEG and MEG measurements. IEEE Trans Biomed butions of EEG and $44(3), 196-208$.

Marchal-Heussler, L., Sirbat, D., Hoffman, M., \& Maincent, P. (1993). Poly(epsilon-caprolactone) nanocapsules in carteolo ophthalmic delivery. Pharm Res, 10(3), 386-390.

Mellanby, J., George, G., Robinson, A., \& Thompson, P. (1977). Epileptiform syndrome in rats produced by injecting tetanus toxin into the hippocampus. I Neurol Neurosurg Psychiatry. $40(4), 404-414$

Nilsen, K.E., Walker, M.C., \& Cock, H.R. (2005). Characterization of the tetanus toxin model of refractory focal neocortica epilepsy in the rat. Epilepsia, 46(2), 179-187.

Paxinos, G., \& Watson, C. (2007). The rat brain in stereotaxic coor dinates (6th ed.). Amsterdam; Boston: Elsevier.

Plummer, C., Harvey, A.S., \& Cook, M. (2008). EEG source localization in focal epilepsy: Where are we now? Epilepsia, $49(2)$ 201-218.

Plummer, C., Wagner, M., Fuchs, M., Harvey, A.S., \& Cook, M.J. (2010). Dipole versus distributed EEG source localization for (2010). Dipole versus distributed EEG source localization for physiol, 27(3), 141-162.
Racine, R.J. (1972). Modification of seizure activity by electrical stimulation. II Motor seizure. Electroencephalogr Clin Neurostimulation. II. Motor seizur
physiol, 32(3), 281-294.

Sander, J.W. (2003). The epidemiology of epilepsy revisited. Curt Opin Neurol, 16(2), 165-170.

Schmidt, D., \& Loscher, W. (2005). Drug resistance in epilepsy: Putative neurobiologic and clinical mechanisms. Epilepsia, Putative neuro
$46(6), 858-877$

Schwartzkroin, P.A., Moshé, S.L., \& Pitkänen, A. (2006). Models of seizures andepilepsy. Amsterdam; Oxford: Elsevier Academic.

Shorvon, S.D. (1996). The epidemiology and treatment of chronic and refractory epilepsy. Epilepsia, 37(Suppl 2), S1-S3.

Tamargo, R.J., Rossell, L.A., Kossoff, E.H., Tyler, B.M., Ewend, M.G., \& Aryanpur, J.J. (2002). The intracerebral administration of phenytoin using controlled-release polymers reduce experimental seizures in rats. Epilepsy Res, 48(3), 145-155.

Traitel, T., Cohen, Y., \& Kost, J. (2000). Characterization of glucosesensitive insulin release systems in simulated in vivo conditions. Biomaterials, 2I(16), 1679-1687.

Varde, N.K., \& Pack, D.W. (2004). Microspheres for controlled release drug delivery. Expert Opin Biol Ther, 4(1), 35-51.

Yeo, Y., \& Park, K. (2004). Control of encapsulation efficiency and initial burst in polymeric microparticle systems. Arch Pharm Res, 27(1), 1-12. 